\title{
Influences of Architectural Shapes of Environment on Electrical Activity of Rat's Brain
}

\author{
M.I. EL-Gohary, E.M. EL-Sayed," G.A. Nagy ${ }^{* *}$, T.M. \\ EL- Sayed and H.M. Hussein \\ Biophysics Branch, Faculty of Science (Boys), Al Azhar University, \\ *Biophysics Branch, Faculty of Science, Ain Shams University, and \\ **High Technology Institute, Tenth of Ramadan City, Sharkia, \\ Egypt.
}

\begin{abstract}
7 THE ARCHITECTURAL shapes of environment may affect the activity of living inhabitants. Accordingly the present work was aimed to study and clarify the influences of architectural shapes of environment on the electrical activity of rat's brain. The brain activities (electroencephalogram, EEG) recorded from rats housed into 2 different models, pyramidal and inverted pyramidal shapes for 3 weeks were studied. The obtained results showed a drop in the mean power amplitude of almost all frequency bands of rat's brain activities placed in pyramidal shape especially of $\theta, \alpha$ and $\beta$ bands. This effect was more pronounced in motor than in visual cortex. On the other hand the recorded rat's brain activities housed in an inverted pyramidal shape showed an enhancement in the mean power amplitude of all frequency bands. The obtained results proved that rats kept in pyramidal shape were more relaxed and more active than that inside an inverted pyramidal environment.
\end{abstract}

Keywords: EEG, Architectural shape, Pyramidal, Inverted pyramidal, Environment.

Many trends appear in architecture aiming to achieve environmental balance and human comfort such as circular, green sustainable architectures and others. Most of these trends have studied the aspects of human comfort as thermal, lighting and noise factors, but they didn't give enough studies to the aspect of human energy balance.

Thus in reality, all matter is in some way connected to the information exchange occurring within the universe, which operates through a variety of frequencies that making up the universe. The matter itself is another form of vibrations at the lowest frequency level leads us to expect that each physical form has a specific set of frequencies "specific patterns" which might influence the human body ${ }^{(1)}$. 
The architecture design of a room may create a new medium that will have an influence on the physical and physiological structure of the bodies. This may be due to bio-energy and the inverse frequencies which may induce similar effects on electromagnetic radiations.

Research on Egyptian pyramids reveals some evidence that the space within the great pyramid and its smaller replicas intensify and/or generate energy of electromagnetic radiations and other forms of the so-called universal energy ${ }^{(2)}$. The effect of this 'pyramidal energy' had been studied on solids, liquids, plants, animals and even human volunteers. Some of the findings for such studies included; rapid growth of plants, faster healing of bruises and burns, longer preservation of milk, an enhanced vitalization and better relaxation of human subjects ${ }^{(3)}$. A number of volunteers have mentioned that meditating inside the pyramid was easier than outside as they felt; more peaceful, more relaxed and less distracted ${ }^{(2,4)}$.

The aim of the present work is to study the influences of different architecture shapes on electrical activity of rat's brain.

\section{Materials}

Male adult albino rats $(n=3 \times 8)$ greater than $150 \mathrm{~g}$ were used in the present study. Animals were kept in animal house under constant laboratory conditions, fed and water ad libitum. The biological clock of the animals was kept as normal.

\section{Experimental System}

The animals were divided into 3 groups (8 for each), the first group considered as control in a wired cage. The other two groups were housed in pyramidal shape of dimensions $21.5 \mathrm{~cm}$ height, $50 \mathrm{~cm}$ base and $22 \mathrm{~cm}$ for each side. The four triangular sides of the pyramidal shape are angled upwards at an angle of $51^{\circ}$ to the base. The inverted pyramidal has a dimension of $25 \mathrm{~cm}$ height, $83 \mathrm{~cm}$ base and $50 \mathrm{~cm}$ side. The four triangular sides of the inverted pyramidal shape are angled upwards at an angle of $129^{\circ}$ to the base. Both geometrical shapes were made of a wood ${ }^{(5)}$ (Fig.1).

\section{Method}

Rats were anesthetized with sodium pentobarbital $(50 \mathrm{mg} / \mathrm{kg}$, intra-peritoneal) prior to electrode implantation. Four epidural permanent stainless electrodes were implanted in the skull in positions overlying both motor and visual cortices of rat's brain.A fifth electrode (reference electrode) was implanted in the contralateral crest of the skull (nasal) at interaural line.

EEG free artifacts signals (120-s) were recorded by the system illustrated in Fig. 2. The activity of left motor cortex area 1 (LMC1), besides the activity of right visual cortex area 17 (RVC17) were simultaneously recorded.

Egypt. J. Biophys. Biomed. Engng. Vol. 12 (2011) 

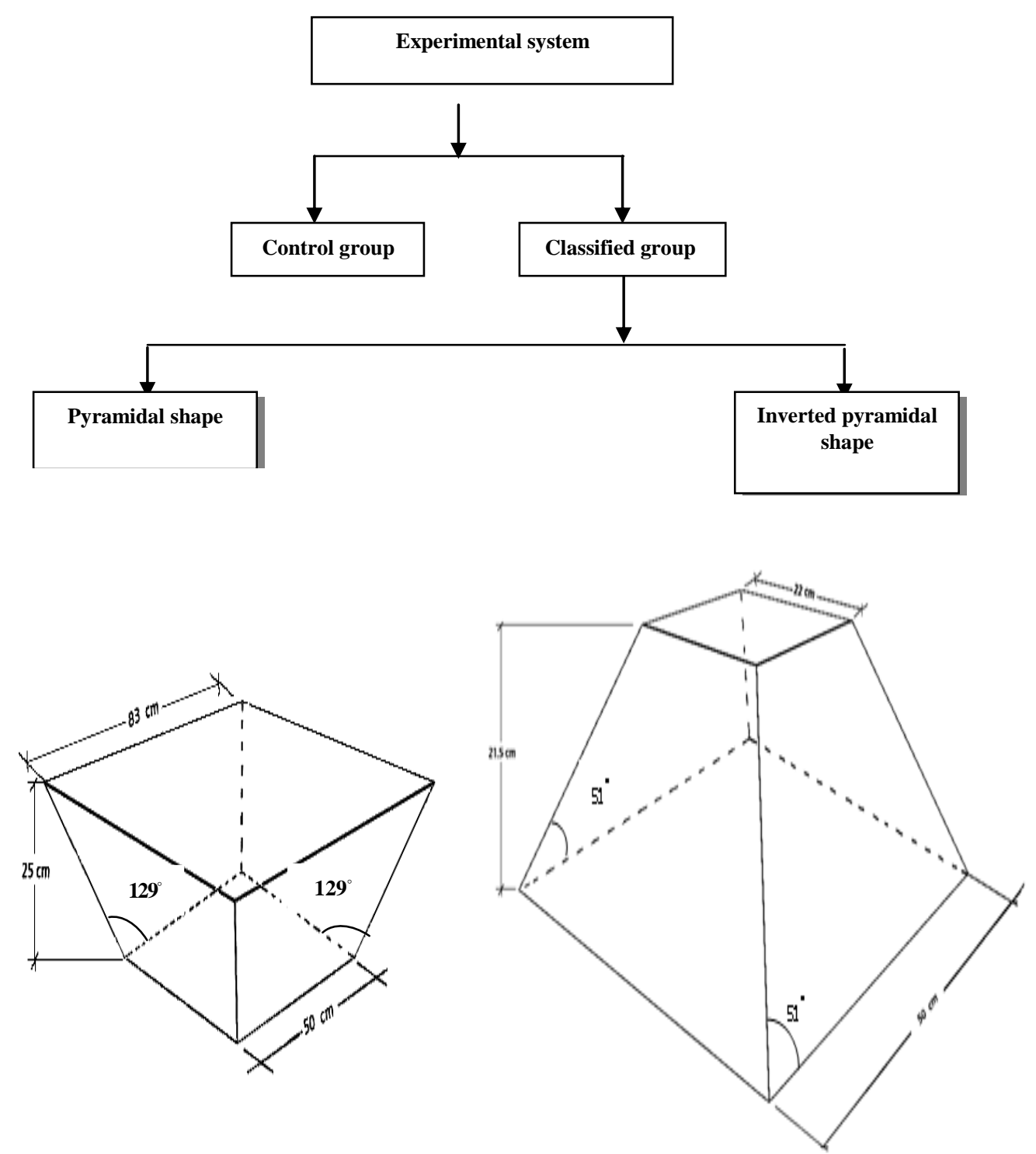

Fig. 1. Charts for housing shapes.

Egypt. J. Biophys. Biomed. Engng. Vol. 12 (2011) 


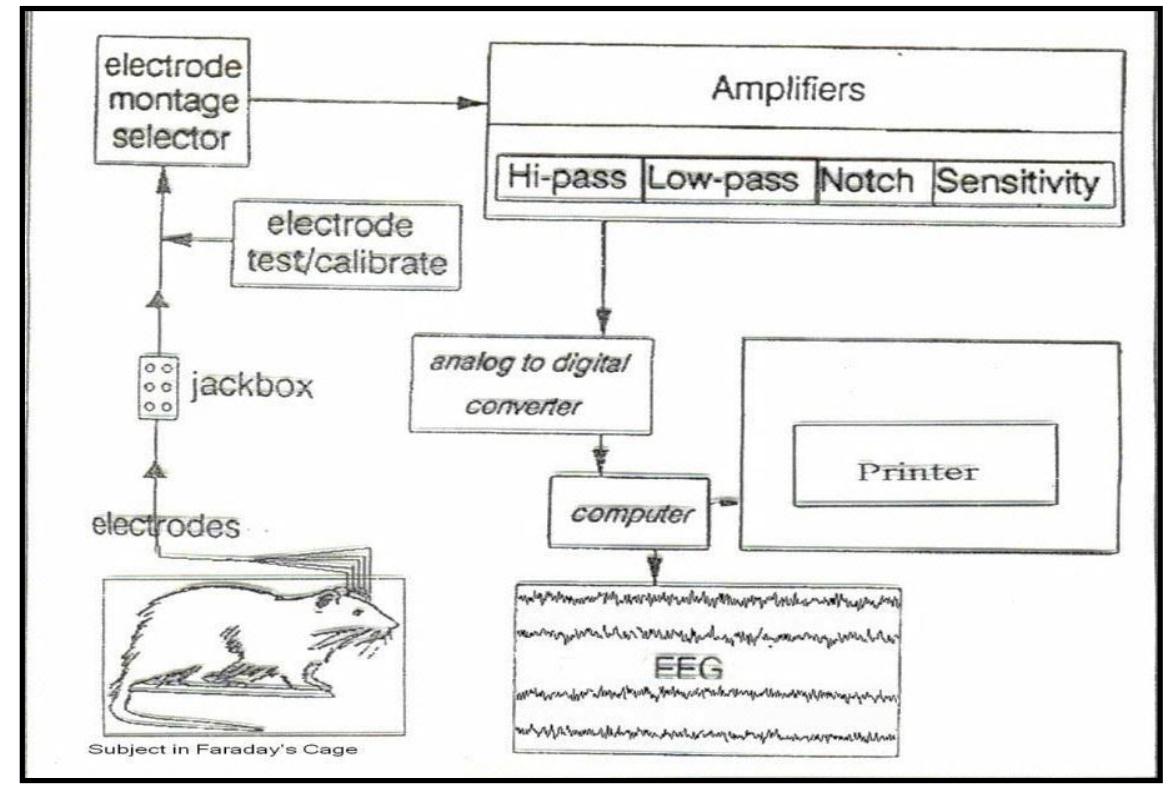

Fig.2. Recording system of EEG signals.

FFT(Fast Fourier Transform)analysis was carried out for EEG signals to create power spectrum relations for the 120-s records. The spectra resulted through this analysis were divided into 4 frequency bands: Delta $(\delta)(1-3 \mathrm{~Hz})$, Theta $(\theta)(4-7 \mathrm{~Hz})$, Alpha $(\alpha)(8-12 \mathrm{~Hz})$ and Beta $(\beta)(13-30 \mathrm{~Hz})^{(6,7)}$. A calculation of mean power of amplitude $\left(\mu \mathrm{V}^{2}\right)$ for EEG signals and standard deviation (S.D) for each band were performed ${ }^{(8)}$.

\section{Results and discussion}

The recorded EEG signals were divided into two main parts based upon the environmental shape; (1) Pyramidal and (2) Inverted pyramidal, and each of them was divided according to the recording area; left motor and right visual cortex.

\section{Pyramidal shape}

Records of EEG signals from left motor cortex

Figure 3 shows the variation of mean power amplitude of different bands of EEG signals recorded from the left rat's motor cortex. After 3 weeks of the environmental housing in the pyramidal shape followed by 2 weeks in ordinary cage, signals were recorded weekly. The EEG records indicate a decrease in the mean power of $\theta$ and $\alpha$ bands, while $\delta$ and $\beta$ nearly stable at control value (Fig. 3). The obtained results showed that mean power amplitude of EEG signals during recovery phase (Recovery.ph.) was fluctuated around control value during the 
first week and then reached control after the second week. These results are in agreement with that obtained by Stark (1977) ${ }^{(9)}$ which showed that, EEG signals from subjects meditating in pyramidal shape has shown higher frequency and higher amplitude of $\alpha$ rhythm. These findings clarify that housing of subjects inside a pyramidal shape caused an improvement of physiological state (relaxation and increment of alertness) ${ }^{(10)}$.

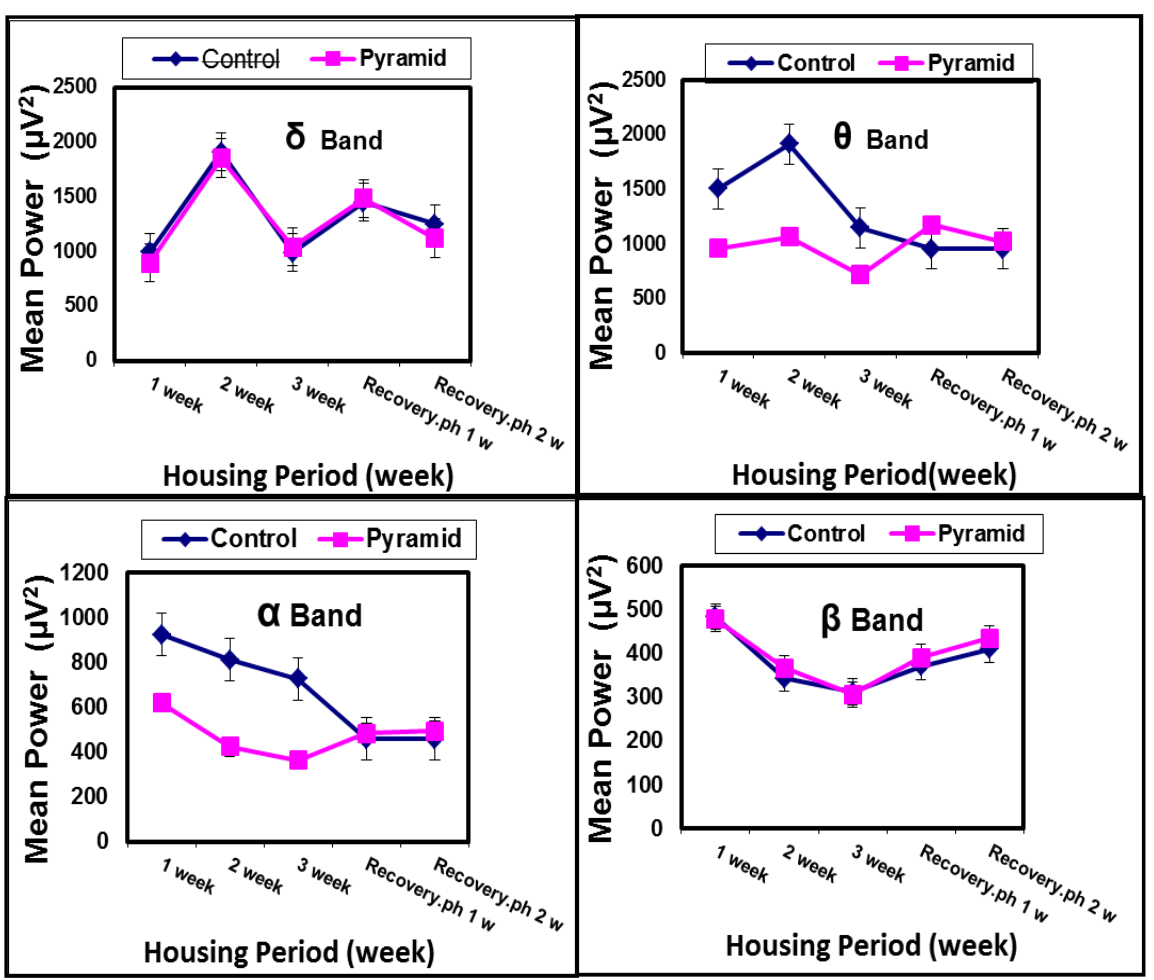

Fig. 3. Mean power amplitude of EEG signals recorded from left motor cortex of rat's brain inside pyramidal shape.

Records of EEG signals from right visual cortex

Figure 4 Illustrates the EEG signals from right visual cortex. It shows an enhancement of the mean power amplitude of all bands except $\theta$. After recovery phase it has reached control value. The decrement of its value for $\theta$ band represents an indication of good mood and this is confirmed by increment of serotonin concentration ${ }^{(11)}$. An increment of $\delta$ band frequency, also showed a relaxation and normal sleep of subjects ${ }^{(12)}$. 


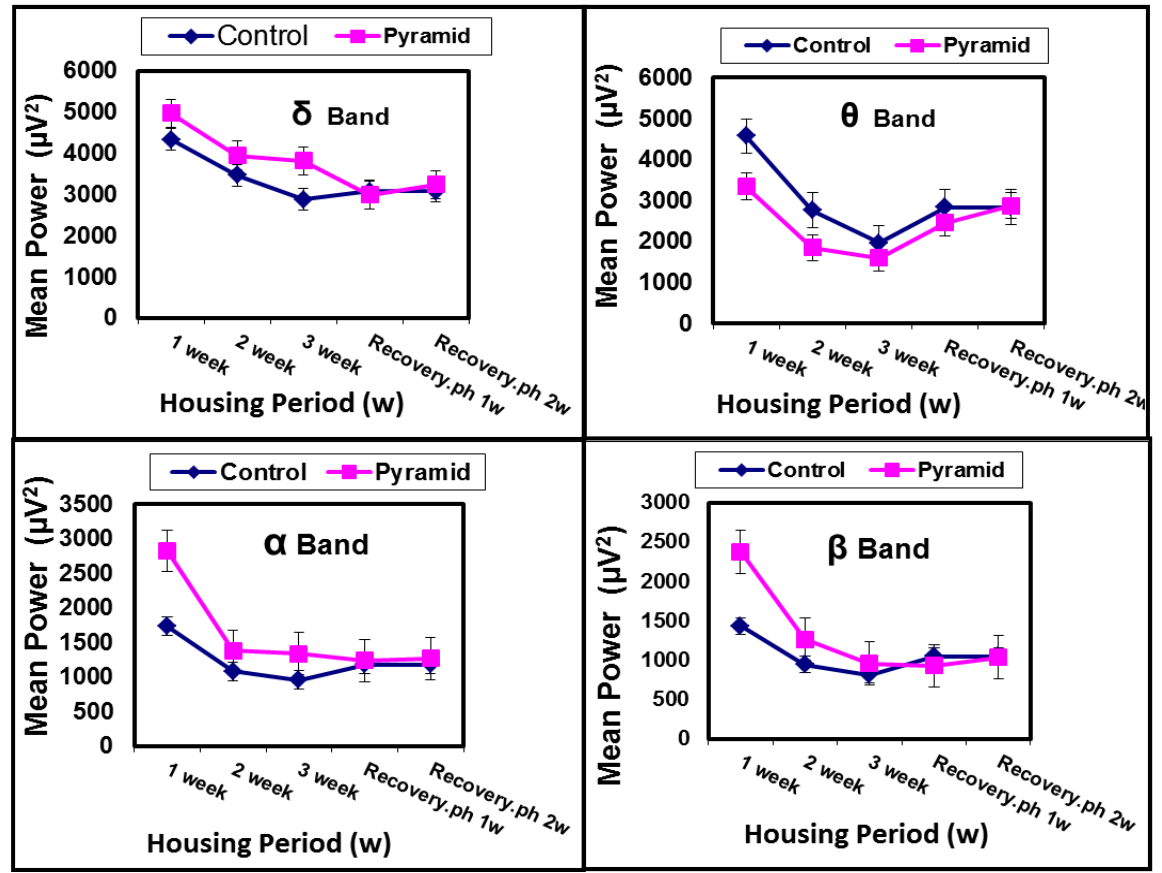

Fig. 4. Mean power amplitude of EEG signals recorded from right visual cortex of rat's brain inside pyramidal shape.

\section{Effect of pyramidal shape on behavior}

Some researchers found that a pyramidal environment counteracted chronic restraint stress-induced neuroendocrine and oxidative stresses in rats ${ }^{(13,14)}$. In the recovery phase after transferring rats from pyramidal shape into normal cage for 2 weeks, the animal suffered from fatigue and irritability.

Frequency shift analysis of EEG signals

In the present part of work, frequency shift analysis of EEG signals was performed in case of pyramidal shape.There was a clear frequency shift towards higher frequency of $\theta$ and $\alpha$ band compared to control. These findings are in agreement with results of $\mathrm{Nagy}^{(1)}$ which proved that pyramidal shape as an environment has an effect on biological systems and its behaviour. This is occurred through an alignment process; as the angle of the Egyptian great pyramid which was aligned with a definite frequency of $7.8 \mathrm{~Hz}$ is nearly same frequency of brain's $\alpha$ band, i.e. resonant frequency.

\section{Inverted pyramidal shape (inv.pyramidal)}

Records of EEG signals from left motor cortex

Figure 5, ilustrates changes of mean power amplitude for different bands of EEG signals recorded from the left motor cortex. After 3 weeks of housing rats in 
an inverted pyramidal shape, an increase in the mean power amplitude of all bands was occurred except for $\delta$ showed a decrement. This reduction in amplitude for $\delta$ band represents transformation of animals into a case of anxiety and sleep disturbance ${ }^{(15)}$. In recovery phase (another 2 weeks inside an ordinary cage),mean power amplitude of EEG signals was oscillated around control and reached its value at the end of the second week.

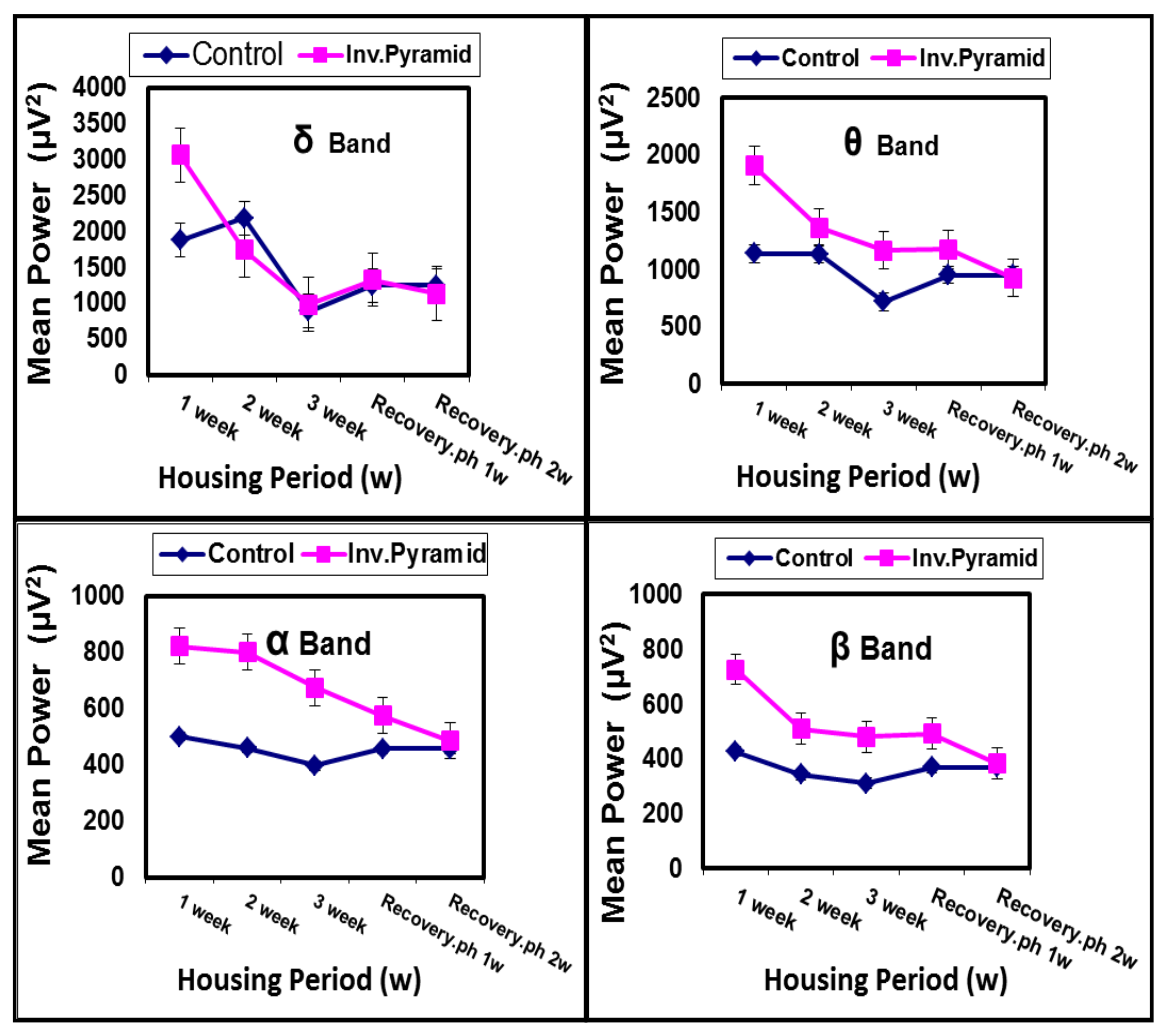

Fig. 5 . Mean power amplitude of EEG signals recorded from the left motor cortex of rat's brain inside an inverted pyramidal shape.

Records of EEG signals from right visual cortex

Figure 6 , explains an enhancement of the mean power amplitude of $\delta$ and drop in $\theta$ band frequency, whereas this parameter for $\alpha$ and $\beta$ bands nearly stable when compared to control. The drop in frequency of $\theta$ band means state of sleep disorders, and drowsiness of animal ${ }^{(16)}$. In recovery phase, values of mean power amplitude for all bands were returned back to control . 


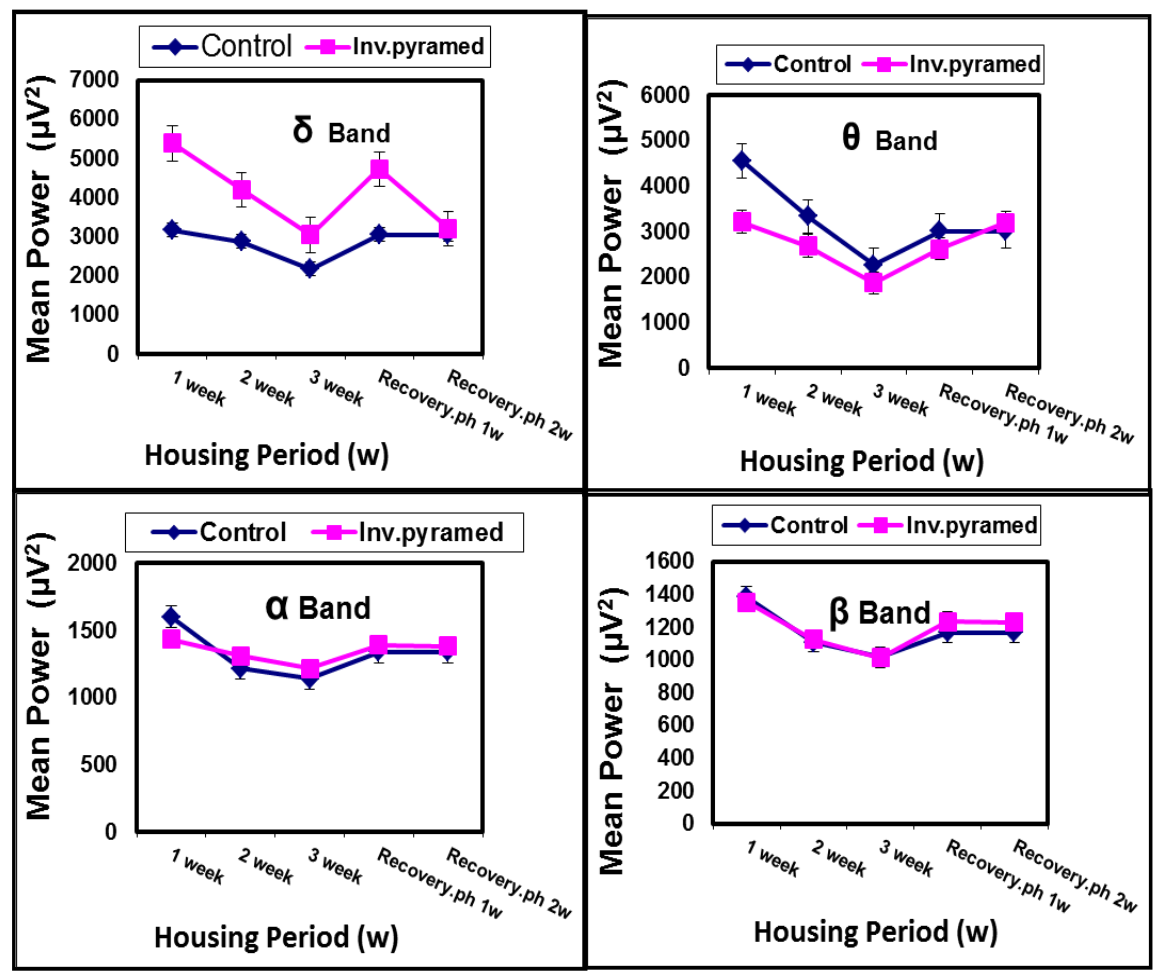

Fig. 6. Mean power amplitude of EEG signals recorded from right visual cortex of rat's brain inside an inverted pyramidal shape. I

Frequency shift analysis of EEG signals

The frequency shift analysis showed that, after 3 weeks inside an inverted pyramidal environment, there was a frequency shift to lower values of $\theta$ and $\beta$ bands, whereas other frequencies of $\delta$ and $\alpha$ bands were unchanged. This means that when rats were kept inside an inverted pyramidal environment a drowsiness of animal and bad mood were observed ${ }^{(17)}$.

\section{Conclusion}

Pyramidal environment

- The reduction in mean power of EEG signals was associated with good mood and normal sleep of subjects. It also showed an enhancement in alertness and relaxation,

- A frequency shift was occurred to higher frequencies in $\alpha$ and $\theta$ bands. This environment has an effect on biological systems and its behavior, and this may be due to an alignment process; as the angle of the Egyptian great pyramid was aligned with frequency $7.8 \mathrm{~Hz}$ which is nearly same 
frequency (resonant frequency) of brains $\alpha$ band. Therefore, mutual transfer of energy has occurred.

An inverted pyramidal environment

- When rats were kept for 3 weeks, this caused an increase in the mean power of EEG signals coupled with a case of drowsiness, anxiety, sleep disorders and depression.

- The placement of rats inside this environment caused frequency shift of EEG signals towards lower frequency of $\theta$ and $\beta$ bands, this led to drowsiness and bad moods of rats.

\section{References}

1. Nagy, G.A. The architectural figuration as a matrix for environmental control due to bioenergy science. Ph. D. Thesis for Department of Architectural Engineering, Faculty of Engineering, Ain Shams University: pp 67-93 (2007) .

2. Schul, B. and Pettit, E. The pyramid: ancient and new miracle worker. In: "The Secret Power of Pyramids", New York: Fawcett Gold Medal. Vol. 3, 11-23 (1975).

3. Gamble, C., "Healing Through Color". Saffrom Woden Thec.W. Danel company (1985).

4. Toth, M. and Nielsen, G. A falcon's eye view of the pyramid. In: "Pyramid Power". USA: Inner Traditions India, pp: 17-23 (1985).

5. Schul, B. and Pettit, E. The historical enigma. In: "The Secret Power of Pyramids", New York: Fawcett Gold Medal, pp: 24-41 (1975).

6. Campbell, I.G. and Feinberg, I. Longitudinal trajectories of non-rapid eye movement delta and theta EEG as indicators of adolescent brain maturation. Proc. Nat. Acad.Sci. U. S. A.Vol. 106, 5177-5180. (2009).

7. Rafael, L., Lars, M., Ernst, M. and Daniel, B. Brain state regulation during normal development: Intrinsic activity fluctuations in simultaneous EEG-fMRI NeuroImage. 60,1426-1439 (2012).

8. Ehlers, C. L., and Havstad, J. W., Characterization of drug effects on the EEG by power spectral band time series analysis. Psychopharmacology Bulletin.. 18, 43-47 (2002).

9. Stark, N.H. What can a pyramid do? In: "The First Practical Pyramid Book", KS: Sheed Andrews and M cMeel Inc., Kansas City. (1977).

10. Barry, R.J., Rushby, J.A., Wallace, M.J., Clarke, A.R., Johnstone, S.J., and Zlojutro I. Caffeine effects on resting-state arousal. Clinical Neurophysiology, 116, 2693-2700. (2005). 
11. El Gohary, M.I., Eraba, Kh.M.T., Gamal, T.M., Boghdady, H.N and El Abiad, N. The influence of microwave irradiation on sleep disorders and electrical activity of rat's brain . Egypt. J. Biophysics and Biomed Eng . 7, pp.28-37. (2006) .

12. Pedley, T. and Daly, D.D. "Current Practice of Clinical Electroencephalography". New Yourk, Raven Press. pp. 1-194. (1990).

13. Surekha, B., Guruprasad, R.A.O., Dilip, K.M. and Gopalakrishna, P. B. Alterations in stress parameter in rats housed in a pyramid model seasonal variation. Int. J. Pharma and Biosciences; 1(2), 1-6 (2010) .

14. Bhat, S., Rao, G., Murthy, K.D. and Bhat, P.G. Housing in pyramid counteracts neuroendocrine and oxidative stress caused by chronic restraint in rats. ECAM, 4(1), 35-42. (2006) .

15. Gamal, T. biophysical effect of cellular phone tower stations on the electrical activity of rats brain. M.Sc. Thesis, Faculty of Science, Al-Azhar Univ., Cairo, Egypt; 86-112. (2002).

16. Maan, M. Sh. EEG waves classifier using wavelet transform and fourier transform. Int. J . Bio. Med Sci. 1(2), 85, 78-87. (2006) .

17. Kotb, H. Sh. The effect of caffeine on the electrical activity of rats brain exposed to magnetic field. M. Sc. Thesis, Faculty of Science, Al-Azhar Univ., Cairo, Egypt; pp 126-145 (2011) .

(Received 9/ 1/ 2013;

accepted $19 / 5 / 2013$ ) 


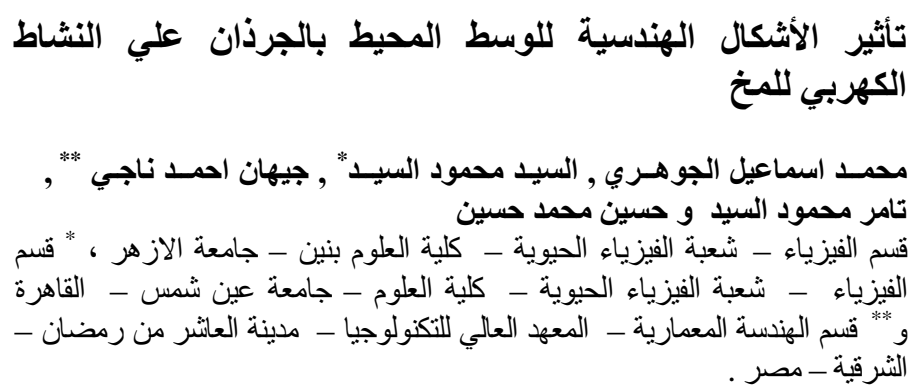

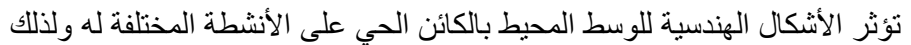

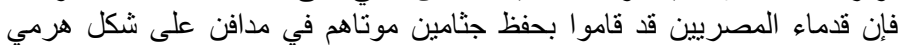

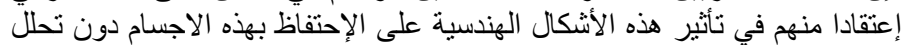

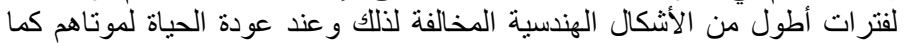

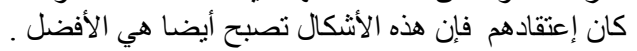

وقد أجري هذا البحث لدراسة تأثير الأشكال الهندسية المختلفة على النشاط

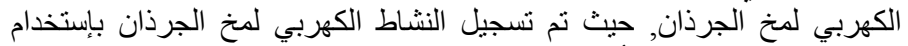

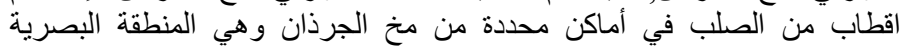

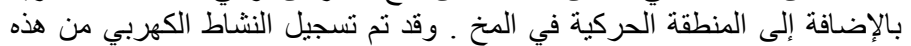

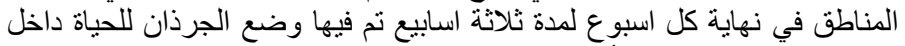

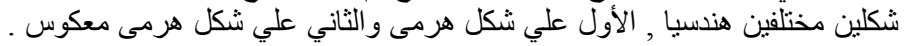

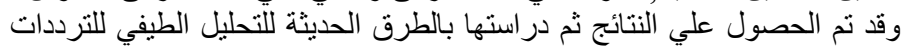

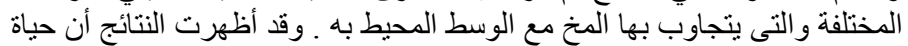

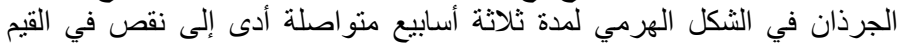

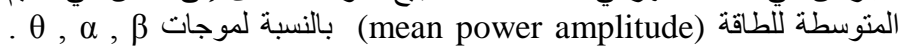

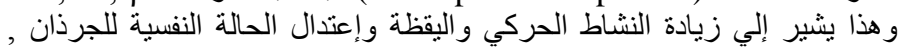

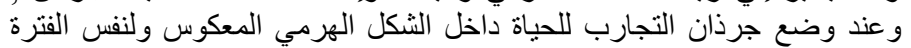

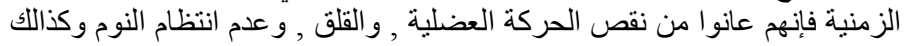

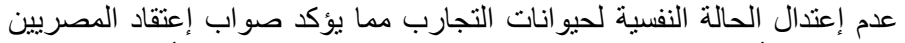

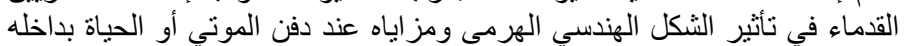

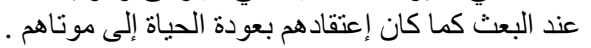

